\title{
Guest Editorial for the Special Issue of ESWEEK 2016
}

Embedded Systems Week (ESWEEK) is the premier event covering major aspects of hardware and software in the design and architecture of embedded and cyber-physical systems. It brings together three leading conferences (CASES, CODES+ISSS, and EMSOFT), two symposia (ESTIMedia and RSP), workshops, and tutorials. With 363 registered attendees, ESWEEK 2016 was well attended, showing the large interest in the field.

ESWEEK 2016 was held in Pittsburgh October 2-7, following the tradition of ESWEEK to rotate between Europe, America, and Asia. At the core of ESWEEK, from Monday to Wednesday, are the three conferences CASES, Codes+ISSS, and EMSOFT, which received 63 technical paper submissions (acceptance ratio 29\%), 80 (26\%), and 98 (26\%), respectively. In addition to the technical paper sessions, special sessions continued to be an important part of the conferences, as experts gave overviews of the newest embedded systems trends. New in 2016 was a focus on the Internet of Things (IoT): The IoT Day (part of Codes+ISSS) presented newest trends in IoT as a mix of technical papers, special sessions, and invited speakers from an embedded-systems point of view. For the first time, the review process of the conferences was conducted in a journal-like, two-stage, peer-reviewed process, with the opportunity for minor/major revision before final decision. This step was in preparation for ESWEEK to move to a journal-integrated publication model starting with 2017.

Highlights of ESWEEK were the three keynote presentations: The Monday keynote by Prof. Srini Devadas from MIT emphasized the importance of Secure Hardware Platforms for the IoT. The Tuesday keynote by Louis K. Scheffer from the Howard Hughes Medical Institute presented new paradigms for how to design software and hardware in Learning from Life. Finally, the Wednesday keynote by Kaushik Roy from Purdue University presented the newest trends in approximate computing, a new paradigm that promises to increase computing efficiency.

Out of the 241 received technical papers, nine outstanding contributions have been nominated as Best Paper Candidates. Finally, the best paper committees selected the best paper for each conference.

The authors of eight papers nominated as Best Paper Candidates for the three ESWEEK conferences have submitted extended versions of their work to this special issue. We have three papers from CASES ("CaffePresso: Accelerating Convolutional Networks on Embedded SoCs," winner of the Best Paper Award; "LOCUS: Low-Power Customizable Many-Core Architecture for Wearables"; and "D-PUF: An Intrinsically Reconfigurable DRAM PUF for Device Authentication and Random Number Generation"), two papers from CODES+ISSS ("Improving Write Performance and Extending Endurance of Object-Based NAND Flash Devices," winner of the Best Paper Award; and "Fault Injection for Test-Driven Development of Robust SoC Firmware"), and three papers from EMSOFT ("Underminer: A Framework for Identifying Nonconverging Behaviors in Black Box System Models," winner of the Best Paper Award; "Simulation-Driven Reachability Using Matrix Measures"; and "Predictable Shared Cache Management for Multicore Real-Time Virtualization").

The CASES papers address three extremely relevant application areas of current and future embedded systems: machine learning, Internet of Things, and support for security. The first paper,

(C) 2017 ACM 1539-9087/2017/11-ART14 \$15.00

https://doi.org/10.1145/3152097 
"CaffePresso: Accelerating Convolutional Networks on Embedded SoCs," proposes new solutions for the implementation of deep learning applications for low-power embedded systems. Specifically, the potential of commercial off-the-shelf hardware for efficient implementation of embedded classification tasks is explored. The authors of "LOCUS: Low-Power Customizable Many-Core Architecture for Wearables" address the issue of modern wearable devices demanding increased computation capability and real-time performance. Under such circumstances, more powerful processors have to be deployed that exceed the power budget available on wearables. The solution proposed in the paper is a customizable many-core architecture that can be deployed on wearable devices independent of the particular application. The problem of secure, low-cost, and robust authentication is addressed in the paper "D-PUF: An Intrinsically Reconfigurable DRAM PUF for Device Authentication and Random Number Generation." The authors propose a solution with Physically Unclonable Functions (PUFs) based on Dynamic Random Access Memories (DRAMs). The key property of DRAMs used for implementing a PUF is their need for periodic refresh operations and the loss of content resulted if the refresh is excessively delayed.

In "Improving Write Performance and Extending Endurance of Object-Based NAND Flash Devices," the authors consider the issue of write amplification in NAND flash memories. Such memories are widely used in low-power embedded systems. However, the fact that an erase operation has to be performed before writing a NAND flash page results in performance and endurance degradation. The solution proposed by the authors is an object-based storage model with a new multilevel garbage collection technique and a selective cache design. In addition to this, a power failure handling technique is employed to guarantee data consistency. The second CODES+ISSS paper, "Fault Injection for Test-Driven Development of Robust SoC Firmware," addresses the issue of growing vulnerability of hardware against soft errors in safety-critical applications. Due to potentially high costs, it is not possible to protect the entire system by hardware mechanisms. Thus, software safety mechanisms have to be deployed and tested. The authors propose solutions to the efficient testing of firmware to ensure the required level of robustness in the presence of soft errors. A host-compiled simulation technique for high-speed fault injection is introduced. Moreover, in order to avoid redundant runs, code analysis is deployed that determines masked and equivalent faults.

Two of the EMSOFT papers address issues related to verification techniques for embedded software. The authors of "Underminer: A Framework for Identifying Nonconverging Behaviors in Black Box System Models" propose a new technique for testing embedded control software. They present a framework for automated checking of stability and convergence of the control system. This is achieved by automatically discovering test inputs that activate potentially nonconvergent behaviors. In order to distinguish between timely convergent and nonconvergent output sequences, the authors also provide a technique to learn the adequate classification functions. The authors of "Simulation-Driven Reachability Using Matrix Measures" propose a technique for simulation-based verification of nonlinear hybrid systems, with strong theoretical guarantees. The technique is based on the construction of safety proofs from simulation data based on an overapproximation of the states reachable from a set of initial states of the system. The paper presents a new technique aimed at reducing the degree of pessimism implied by such an overapproximation. The third EMSOFT paper, "Predictable Shared Cache Management for Multicore Real-Time Virtualization," addresses the issue of running several real-time applications on a single multicore platform using virtualization. A main difficulty in such a context is providing timing predictability. This is due to the sharing of hardware resources, such as memory and communication infrastructure. In this context, the paper presents several solutions to the predictable and efficient management of a shared last-level cache. 
Finally, we would like to thank all authors of the submitted papers and the reviewers who helped put together this special issue on an excellent ESWEEK 2016.

Petru Eles and Jörg Henkel

(Award Chair and General Chair, ESWEEK 2016)

Guest Editors 